\title{
Spatial Bunching of Same-Index Polarization Singularities in Two-Dimensional Random Vector Waves
}

\author{
L. De Angelis, F. Alpeggiani, and L. Kuipers* \\ Kavli Institute of Nanoscience, Delft University of Technology, 2600 GA, Delft, The Netherlands
}

(Received 28 May 2018; revised manuscript received 30 August 2018; published 16 October 2018)

\begin{abstract}
Topological singularities are ubiquitous in many areas of physics. Polarization singularities are locations at which an aspect of the polarization ellipse of light becomes undetermined or degenerate. At $C$ points, the orientation of the ellipse becomes degenerate and light's electric field vector describes a perfect circle in time. In 2D slices of 3D random fields, the distribution in space of the $\mathrm{C}$ points is reminiscent of that of interacting particles. With near-field experiments, we show that when light becomes truly $2 \mathrm{D}$, this has severe consequences for the distribution of $\mathrm{C}$ points in space. The most notable change is that the probability of finding two $\mathrm{C}$ points with the same topological index at a vanishing distance is enhanced in a 2D field. This case is an unusual finding for any system that exhibits topological singularities, as sameindex repulsion is typically observed. All of our experimental findings are supported with theory, and excellent agreement is found between theory and experiment.
\end{abstract}

DOI: 10.1103/PhysRevX.8.041012

\section{INTRODUCTION}

Light-based technology has transformed today's society and will continue to do so, with applications that range from energy harvesting to telecommunications and quantum informatics [1-3]. Increasing control over light's polarization is one key capability inspiring new developments. For instance, optical fields near nanostructures can be engineered to exhibit locations of circular polarization [4-6], allowing applications such as spin-dependent directional coupling [7], also with local solid-state spin into optical information conversion [8]. Interestingly, points of circular polarization are singularities of the light field, also known as $\mathrm{C}$ points $[9,10]$, widely studied in structured light beams [11-13] and representative of the transverse spin momentum of light [14-17].

More in general, $\mathrm{C}$ points are topological defects of the vector field which describes light's polarization. Knowledge and study of topological defects goes way beyond optics. Currently, dislocations of the local magnetization known as Skyrmions are being intensively investigated [18-20]. In nematic systems, topological defects have continuously attracted interest because of their fascinating behavior $[21,22]$. In addition, these kinds of defects can even govern the physics of biological systems [23], and their spatial arrangement is representative

\section{*1.kuipers@tudelft.nl}

Published by the American Physical Society under the terms of the Creative Commons Attribution 4.0 International license. Further distribution of this work must maintain attribution to the author(s) and the published article's title, journal citation, and DOI.
Subject Areas: Optics, Statistical Physics

of intrinsic properties of the system in which they are found [24].

Interestingly, the large ensemble of $\mathrm{C}$ points which naturally arises in random light fields also exhibits an emblematic and rigorous spatial distribution [25-28], which resembles that of particles in a simple liquid and only scales with the wavelength of the interfering waves [26]. However, a random wave field can be realized in several ways [29-36]. So far, the work has concentrated on the investigation of polarization singularities in twodimensional (2D) slices through random three-dimensional (3D) fields in the paraxial limit. The question now arises as to how limiting the propagation of light to a truly $2 \mathrm{D}$ situation, e.g., by confining it on a flat optical chip, would be to the spatial distribution of its polarization singularities. In such a case, transverse propagation would set a one-to-one relation between the wave propagation direction and the direction of the electric field. Moreover, this would create correlations between right-handed and left-handed polarization that are absent in the threedimensional fields.

By means of near-field experiments, we investigate the spatial distribution of $\mathrm{C}$ points in a planar random light field and reveal crucial differences with respect to existing paraxial theory [26]. We demonstrate that confining light propagation in two dimensions leads to a large increase in the probability of finding, at close proximity, $\mathrm{C}$ points with the same topological charge, i.e., their index. This is an exotic behavior for topological singularities, which usually exhibit same-charge repulsion. We relate our experimental findings to light's handedness and excellently describe them with a new theoretical model developed for the two-dimensional case. 
(a)

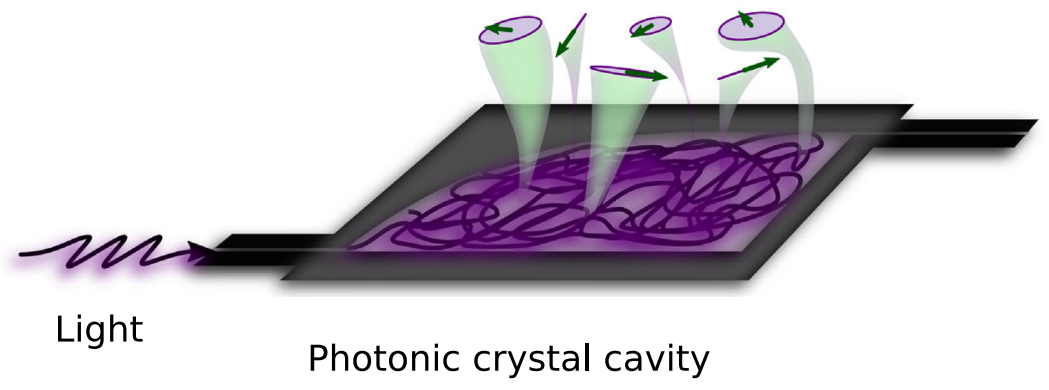

(b)

Polarization ellipse

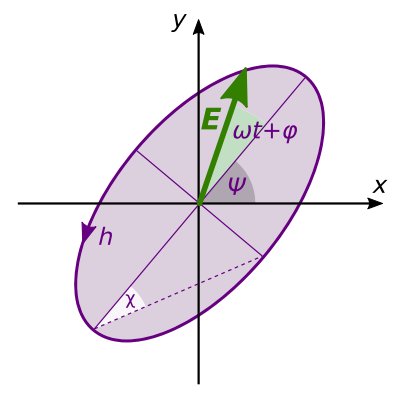

(c)

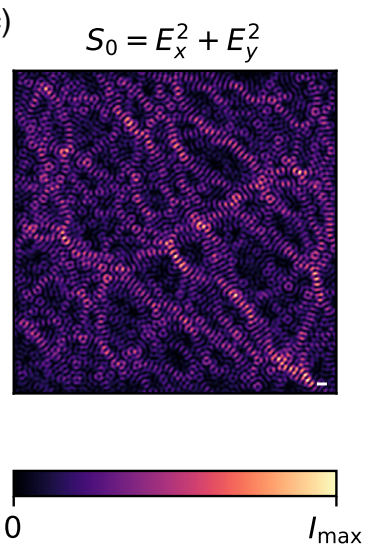

(d)

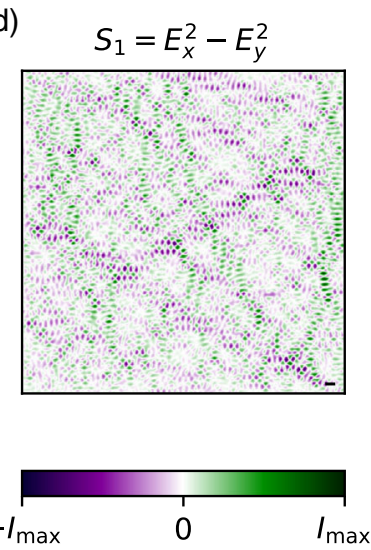

(e)

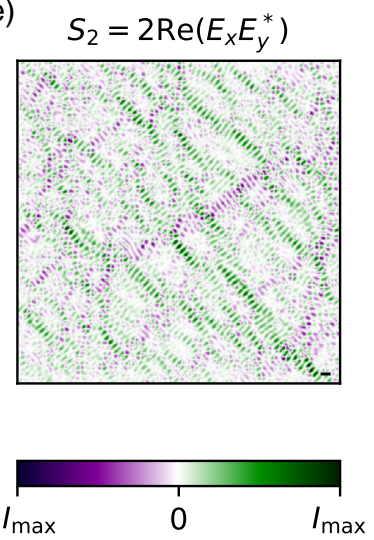

(f)
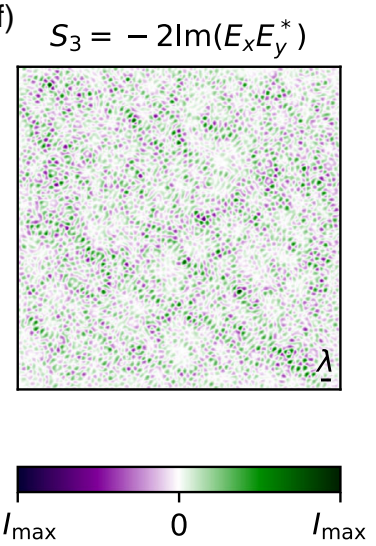

FIG. 1. Overview of the near-field measurements of light's polarization in a chaotic cavity. (a) Schematic of the experimental realization of random light waves in the planar photonic crystal cavity (black). Inside the cavity, light exhibits a spatially dependent polarization. This is illustrated by a few different polarization ellipses (purple ellipses) observed at different points in the cavity (indicated by the green shadows). (b) Parametrization of the polarization ellipse, describing the local polarization state of light. (c)-(f) Near-field maps of the Stokes parameters of the optical random field in a square region of $17 \mu \mathrm{m} \times 17 \mu \mathrm{m}$ inside the chaotic cavity. Note that $S_{0}=E_{x}^{2}+E_{y}^{2}$ is the total intensity of the vector field. This is displayed with a false-color map ranging from 0 to $I_{\max }$, where $I_{\max }$ is the maximum measured intensity. Here, $S_{i}(i=1,2,3)$ describes the polarization state of light, with respect to linear (horizontalvertical), linear ( $\pm 45 \mathrm{deg}$ ), and circular (right-left) polarizations, respectively. These Stokes parameters are also represented with falsecolor maps, which this time range from $-I_{\max }$ to $I_{\max }$.

\section{EXPERIMENT AND METHODS}

\section{A. Near-field optical measurements}

In our experiments, we map the near field of light waves propagating in the planar chaotic cavity sketched in Fig. 1(a). This is a photonic crystal cavity realized in a siliconon-insulator platform (220-nm silicon slab) and designed to provide random wave propagation [37]. With a monochromatic laser at the telecom frequencies $\left(\lambda_{0} \simeq 1550 \mathrm{~nm}\right)$, we excite a transverse electric (TE) slab mode, which results in a random superposition of monochromatic TE waves inside the cavity $[38,39]$. With a custom-built near-field scanning optical microscope (NSOM), we probe the light field approximately $20 \mathrm{~nm}$ above the surface of the cavity. The measurement of the amplitude and phase of both the in-plane components $\left(E_{x}, E_{y}\right)$ allows the full characterization of its polarization state at every point in the measured plane. For simplicity, we only consider the TE light propagating in the sample, which has its electric field entirely in the plane of propagation. We do not investigate TM light, as our cavity was not designed to confine it [37].

A comprehensive description of light's polarization is provided by its Stokes parameters [40]. These parameters are often used to characterize the polarization state of light in the far field, ranging from a simple laser beam to the polarized emission of exotic structures [41], but they can be used for a local analysis of the near field as well. Figures 1(c)-1(f) present the near-field maps of the Stokes parameters for the optical random field inside the chaotic cavity. As a result of vector light waves randomly interfering, these patterns are quite difficult to interpret. However, we can spot a few specific features in the morphology of each map. Note that $S_{1}$ exhibits patterns of spatial modulation approximately half a wavelength wide and several wavelengths long. Depending on their color (sign), these stripy patterns are either oriented along the $x$ or the $y$ axis. 
The same observation is valid for $S_{2}$, but here the modulations are oriented at \pm 45 deg with respect to the horizontal axis. No clear preferential direction stands out from the map of $S_{3}$. In fact, $S_{1}$ is representative of light linearly polarized along $x\left(S_{1}>0\right)$ or $y\left(S_{1}<0\right)$, and since light propagates as a transverse vector wave, the observed stripy patterns are reminiscent of $x$-polarized waves mainly propagating along $y$ and vice versa [38]. A totally analogous argument holds for $S_{2}$, while $S_{3}$ does not exhibit any pattern that bears a resemblance to any specific in-plane direction since it is the parameter representative of circular polarization.

\section{B. Light's polarization and $\mathrm{C}$ points}

A more concise, yet comprehensive summary on the complex polarization pattern illustrated in Fig. 1 can be obtained from the analysis of its singularities [28]. In general, light's polarization is elliptical and thus parametrized with the orientation $\psi$ of the polarization ellipse, the ellipticity angle $\chi$, and the handedness $h$ [Fig. 1(b)]. However, there are special cases in which the polarization ellipse degenerates into a circle or a line, and some of these parameters are not well defined anymore. In two dimensions, such singularities of the vector field are, respectively, points of circular polarization (C points) and lines of linearly polarized light (L lines) [42].

Figure 2 is a map of the orientation of the polarization ellipse for a small subsection of the measurement presented in Fig. 1. The position of $\mathrm{C}$ points is highlighted by circles and triangles, whose color represents their topological index $I$. This is defined as the half-integer number of

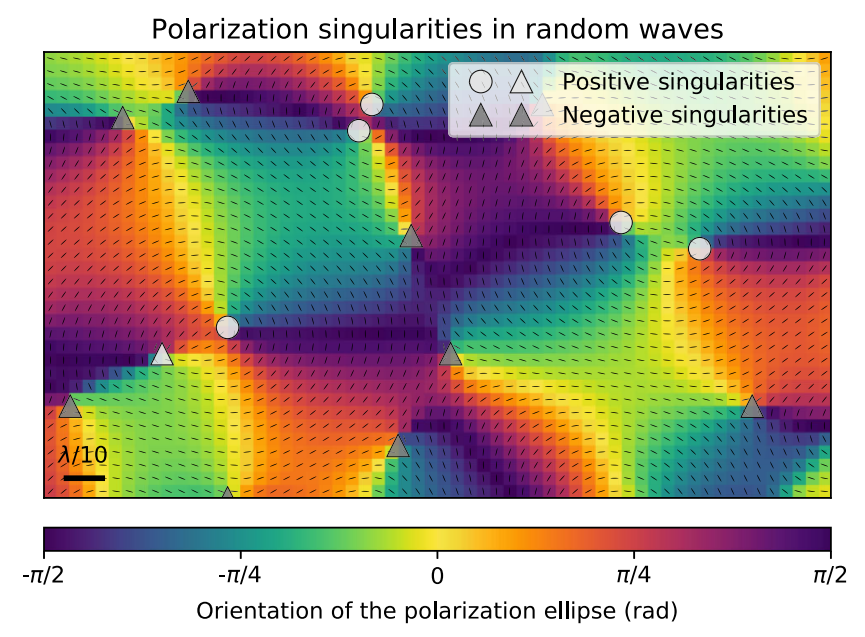

FIG. 2. False-color map for the orientation of the major axis of the polarization ellipse. The black directors indicate the orientation of such an axis, too. The plot is representative of a subsection of the measured optical random field. Circles and triangles are $\mathrm{C}$ points. The white and gray symbols denote positive and negative topological indices, respectively. The shape of the symbols, triangles or circles, denotes a star-type or lemon-type classification, respectively. times that the axis of the polarization ellipse rotates around the singularity, clockwise (positive index) or anticlockwise (negative index), i.e.,

$$
I=\frac{1}{2 \pi} \int_{\mathcal{C}} \mathrm{d} \psi
$$

where $\mathcal{C}$ is a closed path enclosing one singularity. In Fig. 2, we only observe topological indices of $\pm 1 / 2$.

Strictly related to their topological index is the so-called line classification of $\mathrm{C}$ points, which differentiates them into three types: lemons, stars, and monstars $[43,44]$. The line classification can be understood by looking at the orientation of the polarization ellipse around the singularity, highlighted by the black directors in Fig. 2 and in the zoomed-in images of Fig. 3. For lemon-type singularities (lemons), there is only one direction along which the orientation of the polarization ellipse is directed towards the singularity, whereas the possible directions are always three for star-type singularities (stars and monstars). To determine the line classification of all the $\mathrm{C}$ points in our data set in a deterministic way, we apply the method illustrated by Dennis for computing the number of directors pointing towards each singularity [43]. In our figures, we indicate stars and monstars with triangles, and lemons with circles.

Already, a quick glance at Fig. 2 illustrates the clear relation between the topological index (marker color) and line classification (marker shape) of $\mathrm{C}$ points. In fact, negative-index singularities are always stars, whereas both lemons and monstars are characterized by a positive index, as expected, in general, for C points [43]. Table I lists the fraction of $\mathrm{C}$ points for each kind observed in our experimental data set. Note that $50 \%$ of the total number of $\mathrm{C}$ points are stars, and they all carry a negative topological index. Approximately $45 \%$ of the singularities are lemons, and only 5\% monstars, both types exhibiting a positive index. In the same table, we directly compare our experimental outcome with the results from previous paraxial theory [26] and experiments [27]. All of these examined statistics are perfectly consistent with each other. In summary, the abundance of $\mathrm{C}$ points with a particular line
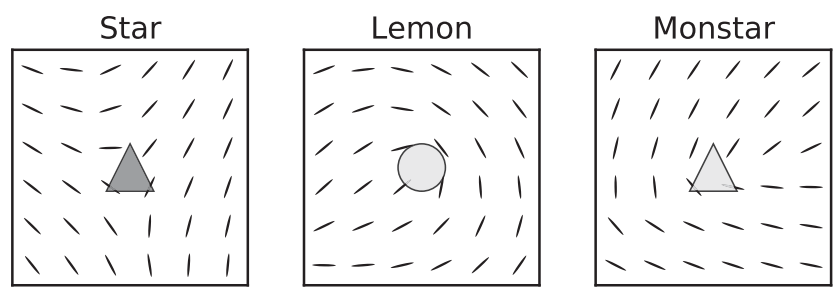

FIG. 3. An overview of the three kinds of $\mathrm{C}$ points based on their line classification [43]. The lines are the orientation of the polarization ellipse at each pixel around the $\mathrm{C}$ point (circle or triangle), as determined from experimental data. 
TABLE I. Fraction of $\mathrm{C}$ points with different line classification. The results of our $2 \mathrm{D}$ experiment are compared with previous experiments [27] and theory [26].

\begin{tabular}{lccc}
\hline \hline $\begin{array}{l}\text { Singularity } \\
\text { type }\end{array}$ & 2D field & \multicolumn{2}{c}{ 2D slice of a 3D field } \\
\hline Star & $0.4997 \pm 0.0002$ & $0.506 \pm 0.003$ & 0.500 \\
Lemon & $0.4493 \pm 0.0013$ & $0.443 \pm 0.002$ & 0.447 \\
Monstar & $0.0503 \pm 0.0013$ & $0.050 \pm 0.003$ & 0.053 \\
\hline \hline
\end{tabular}

classification is the same for $\mathrm{C}$ points in truly twodimensional light and two-dimensional slices through a three-dimensional field.

\section{SPATIAL DISTRIBUTION OF C POINTS}

\section{A. Pair and charge correlation function}

Having established that there is no difference between the abundances of the various types of singularities observed in 2D slices of 3D light fields and truly 2D fields, the question now arises as to whether their distribution in space is also the same. The natural way of investigating the spatial distribution of pointlike singularities is by determining their pair correlation function $g(r)$. Given a $\mathrm{C}$ point, this function describes how the density of the surrounding $\mathrm{C}$ points varies as a function of distance. This method is widely used to describe the physics of discrete systems [45-50], it can be directly related to the structure factor [51], and it represents a spatial analogous of the degree of second-order coherence $g^{(2)}(\tau)$, commonly used to determine photon bunching and antibunching [52].

Figure 4 presents the pair correlation function for $\mathrm{C}$ points in two-dimensional random light, as obtained from

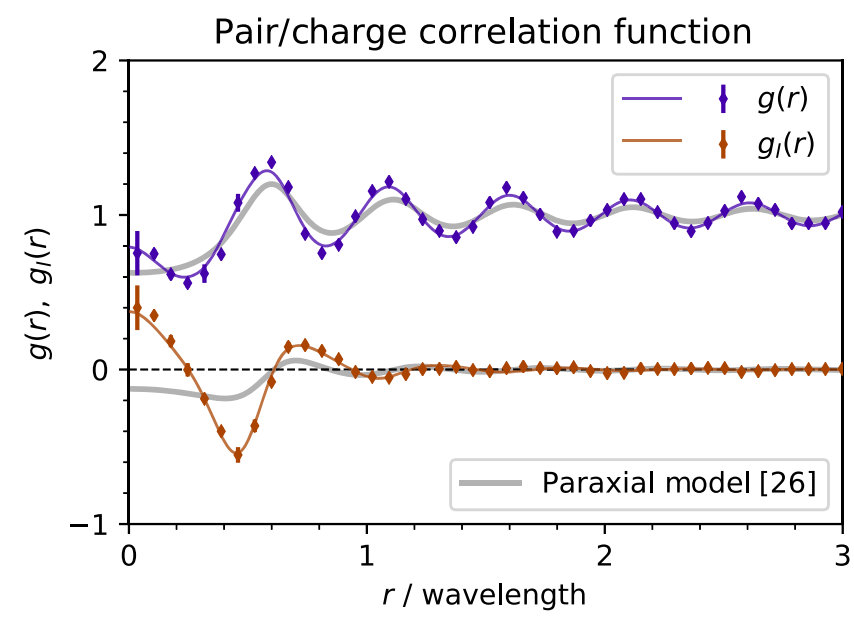

FIG. 4. Pair and charge correlation function $\left(g, g_{I}\right)$ for $\mathrm{C}$ points in random vector waves. The circles are the experimental results, blue and yellow solid lines show our model for 2D vector fields, and gray lines are the paraxial model [26]. our experimental data. With the position of each singularity known, we can compute their pairwise distances $\left|\mathbf{r}_{i}-\mathbf{r}_{j}\right|$, and eventually the pair correlation function

$$
g(r)=\frac{1}{N \rho}\left\langle\sum_{i \neq j} \delta\left(r-\left|\mathbf{r}_{i}-\mathbf{r}_{j}\right|\right)\right\rangle,
$$

where $N$ is the total number of singularities, $\rho$ is the average density of surrounding singularities, and $\delta$ is the Dirac function. We compute the average and uncertainty of such a correlation function by combining the outcome of 20 nearfield measurements of the optical random field under investigation. In each of these maps, we precisely pinpoint the location and topological index of approximately $6500 \mathrm{C}$ points, with a spatial accuracy that is limited by the pixel size of the experiment $(\approx 20 \mathrm{~nm})$.

Note that $g(r)$ is not flat, indicating that $\mathrm{C}$ points in random light exhibit spatial correlation. At first glance, this $g(r)$ seems similar to the one of phase singularities in scalar random waves $[38,53]$ and therefore also reminiscent of that of particles in a simple liquid. In fact, $g(r)$ displays a damped oscillatory behavior around unity as a function of $r$, with a maximum, representative of a surplus of singularities, at approximately half a wavelength of distance. Surprisingly, the pair correlation of $\mathrm{C}$ points in 2D actually increases as $r$ approaches 0 . While the zero dimensionality of optical singularities would, in principle, allow for a finite probability of having two at the same location, an increase of $g(r)$ towards zero has never been observed, neither for phase singularities in scalar or vector random waves $[38,53]$ nor for $\mathrm{C}$ points in a $2 \mathrm{D}$ slice of a $3 \mathrm{D}$ random field (Ref. [26] and gray lines in Fig. 4).

To understand the unexpected behavior at small distances and to obtain an overview of the spatial distribution of the $\mathrm{C}$ points, it is useful to also consider the charge correlation function $g_{I}(r)$ : a more general expression of the pair correlation function in which each singularity is weighted with its topological index [26,53]. The orange data points in Fig. 4 display our experimental results for $g_{I}(r)$. The most striking observation here is that the charge correlation function is positive near $r=0$. This means that when singularities are found at a close distance from each other, they most often carry the same topological index. Then, at $r \approx \lambda / 4$, the charge correlation function flips sign, indicating the beginning of a displacement range where two singularities are more likely to have opposite sign. The zero crossing roughly coincides with the distance at which $g(r)$ exhibits the unexpected increase towards small $r$. This increase can therefore be attributed to the surplus of samesign singularities in such a displacement range.

The reason why $\mathrm{C}$ points in $2 \mathrm{D}$ tend to rearrange to form closely spaced pairs with the same topological index is, at this stage, still unclear. The origin of the clustering should be sought in correlations among different field components set by the modal properties of the field. However, the 
topological index is not the only intrinsic property carried by $\mathrm{C}$ points. We could gain more insight by analyzing their behavior with respect to light's handedness.

\section{B. C points and light's handedness}

The correlation functions displayed in Fig. 4 provide an extensive description of the distribution of $\mathrm{C}$ points but still not the full picture. This is because the information carried by $\mathrm{C}$ points is not limited to their topological index. In fact, light's polarization is purely circular at every $\mathrm{C}$ point; however, it can be left- or right-handed, independent of the topological index. In Fig. 5, we show a spatial map of the degree of circular polarization $s_{3}=S_{3} / S_{0}$, together with the position, topological index, and handedness of the $\mathrm{C}$ points therein. We notice how $\mathrm{C}$ points fall in domains of a given handedness. Of course, $s_{3}$ equals exactly +1 or -1 at every $\mathrm{C}$ point, with a sign that determines the handedness of the $\mathrm{C}$ point itself. Each domain is delimited by $\mathrm{L}$ lines (white lines), where polarization is purely linear $\left(s_{3}=0\right)$, and light's handedness is undetermined. L lines have to separate C points of opposite handedness. Contrarily, several co-handed singularities can occur within the same domain. Furthermore, from Fig. 5, one immediately realizes how the handedness and topological index of a $\mathrm{C}$ point are not directly related, as every combination of these quantities is possible.

The handedness of $\mathrm{C}$ points provides an additional degree of freedom, to be accounted for in their spatial distribution. It is illuminating to include this degree of freedom in the computation of a new set of pair correlation functions. In general, $g(r)$ can be expressed as the average of all possible partial correlation functions for $\mathrm{C}$ points with

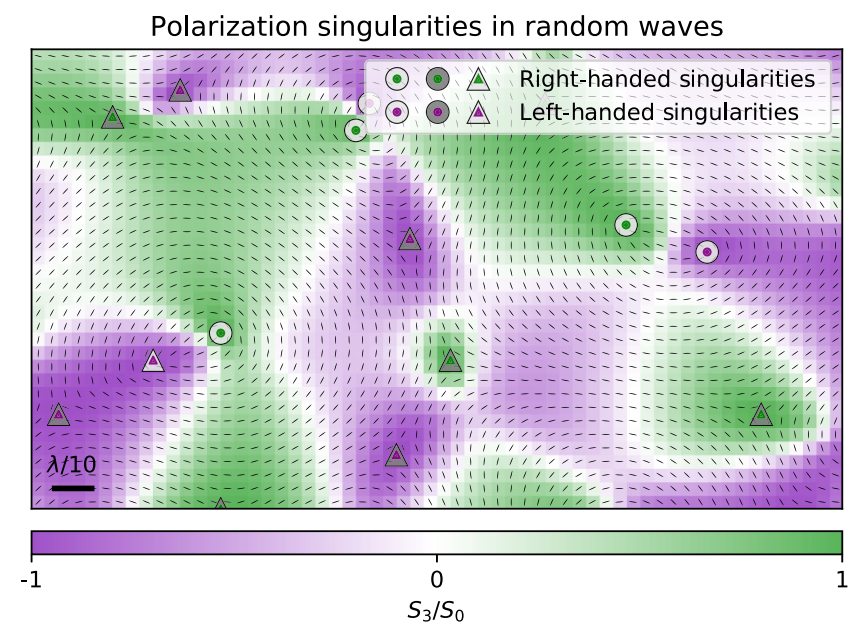

FIG. 5. False-color map for the degree of circular polarization $s_{3}=S_{3} / S_{0}$, as obtained from our experimental data. The plot corresponds to the same subsection of the measured optical random field displayed in Fig. 2. The black directors indicate the orientation of the polarization ellipse. Circles and triangles are $\mathrm{C}$ points, and their color (purple or green) represents their handedness (left or right, respectively). the same or opposite handedness and the same or opposite topological index:

$$
g(r)=\frac{1}{16} \sum_{i, j} \sum_{\alpha, \beta} g_{i, j}^{\alpha, \beta}(r),
$$

with $i, j \in[+,-]$ for the topological index and $\alpha, \beta \in[l, r]$ for the handedness. Following the notation of Dennis [26], Eq. (3) can be simplified with the definitions

$$
g_{\text {same }}^{C} \equiv g_{i, i}^{\alpha, \alpha} \quad \text { and } \quad g_{\text {opp }}^{C} \equiv g_{i,-i}^{\alpha, \alpha},
$$

both corresponding to co-handed singularities, for the cases of the same and opposite topological indices, respectively. Analogously, for antihanded $\mathrm{C}$ points, we have

$$
g_{\text {same }}^{A} \equiv g_{i, i}^{\alpha, \bar{\alpha}} \quad \text { and } \quad g_{\text {opp }}^{A} \equiv g_{i,-i}^{\alpha, \bar{\alpha}} .
$$

Thus, we can express Eq. (3) as a function of these four correlation functions:

$$
g(r)=\frac{1}{4}\left[g_{\mathrm{same}}^{C}+g_{\mathrm{opp}}^{C}+g_{\mathrm{same}}^{A}+g_{\mathrm{opp}}^{A}\right] .
$$

Figure 6 presents our experimental results for the four pair correlation functions of the decomposition in Eq. (6), taking both the topological index and handedness of the $\mathrm{C}$ points into account. In the distribution functions depicted in Fig. 6(a), we only consider co-handed C points, either with the same (green) or opposite (purple) topological indices. In this case, the experimentally determined functions describe the standard characteristic properties exhibited by phase singularities in random waves. In fact, $g_{\text {same }}^{C}(r \rightarrow 0)=0$ for singularities with the same topological index, and there is a monotone decrease towards a finite value at $r \rightarrow 0$ in $g_{\mathrm{opp}}^{C}$. The experimental results displayed in Fig. 6(a) perfectly match the prediction of the model for polarization singularities in a $2 \mathrm{D}$ slice of a 3D field in the paraxial regime [26], which is equivalent to the model for phase singularities in scalar random waves [53].

In fact, we can interpret $C$ points as phase singularities in either the left- or right-handed circular components of $\mathbf{E}$ :

$$
\psi_{l}=E_{x}+i E_{y}, \quad \psi_{r}=E_{x}-i E_{y} .
$$

This is because a phase singularity in $\psi_{l}$ corresponds to a zero in $\psi_{l}$, resulting in a point where $\mathbf{E}$ only has contributions from its circular-right component $\psi_{r}$, i.e., a right-handed $\mathrm{C}$ point, and vice versa. Therefore, the spatial distribution of co-handed $\mathrm{C}$ points is exactly equivalent to that of phase singularities arising in a single circular field component $\psi_{l / r}$, i.e., of phase singularities in a scalar random wave field [26].

Our experiment confirms that, also in 2D, the distribution of co-handed $\mathrm{C}$ points is the same as that of phase 

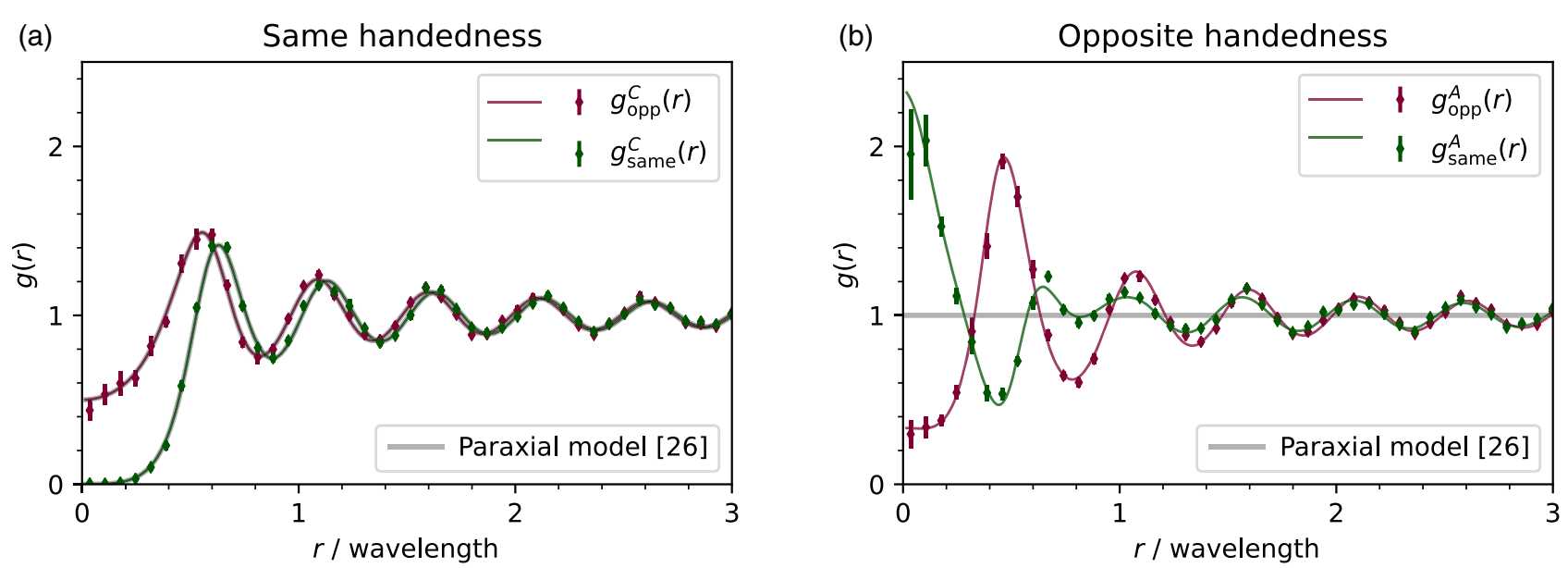

FIG. 6. Pair correlation function $g(r)$ for C points with the same (a) or opposite (b) handedness, and the same $\left(g_{\text {same }}\right)$ or opposite $\left(g_{\text {opp }}\right)$ topological index. Data points represent our experimental results, colored solid lines show our model for an isotropic $2 \mathrm{D}$ random field, and solid gray lines are the 3D paraxial model [26]. The solid gray lines in panel (a) overlap exactly with the colored solid lines.

singularities in a scalar random field. Therefore, the origin of the unusual behavior of the global distribution of $\mathrm{C}$ points must necessarily lie in antihanded singularities. Figure 6(b) presents the correlation functions for singularities with opposite handedness. Here, $g_{\text {same }}^{A}(r)$ reaches its maximum values at $r \approx 0$. Singularities of opposite handedness and the same topological index are often found at close distances from each other, confined in an extremely subwavelength regime. Regarding pairs of $\mathrm{C}$ points with opposite topological index, the distribution $g_{\text {opp }}^{A}$ exhibits a behavior that is qualitatively very similar to that of $g_{\mathrm{opp}}^{C}$. This result creates two clearly distinct behaviors for the four combinations of index and handedness. On the one hand, the impact of the handedness of $\mathrm{C}$ points on their spatial correlations seems to be only minor for singularities with opposite topological indices, for which we do not observe big qualitative differences between $g_{\mathrm{opp}}^{C}$ and $g_{\mathrm{opp}}^{A}$ (purple data in Fig. 6). On the other hand, considering the same or opposite handedness is crucial in the same-index case, since the behaviors of $g_{\text {same }}^{C}$ and $g_{\text {same }}^{A}$ are evidently different, eventually with an opposite gradient for $r \rightarrow 0$ (green data in Fig. 6).

As a matter of fact, the data displayed in Fig. 6(b) offer a clear illustration of the novel behavior registered for $\mathrm{C}$ points in $2 \mathrm{D}$ random light compared to the case of a $2 \mathrm{D}$ slice of a 3D field. In particular, it clarifies that, in the 2D case, $\mathrm{C}$ points of opposite handedness are far from being independent and so must be for the left- and right-handed field projections from which they arise.

\section{CORRELATION AMONG LIGHT'S VECTOR COMPONENTS}

The overall spatial correlation of $\mathrm{C}$ points in 2D random light (Fig. 4) and, more specifically, the correlation of singularities with opposite handedness [Fig. 6(b)] exhibit a number of features that were not accounted for in a previous paraxial theory [26]. In that theory, an assumption was made, consisting of the absence of any correlation between oppositely handed $\mathrm{C}$ points, i.e., $g_{\mathrm{same}}^{A}=g_{\mathrm{opp}}^{A}=1$. This assumption corresponds to a situation in which $\psi_{l}$ and $\psi_{r}$ are completely uncorrelated.

In fact, in three dimensions, there are no restrictions that would imply a correlation among the circular components $\psi_{l}$ and $\psi_{r}$ of a paraxial random field. The same holds true for a two-dimensional slice of such a three-dimensional field [27]. In this circumstance, transversality can be fulfilled out of the plane in which the field is observed, meaning that the vector components of such a field can even be independently generated. Contrarily, in a truly two-dimensional vector field, transverse propagation must be fulfilled in the same plane in which the waves are actually propagating. Dismissing the third dimension while obeying transversality then results in a correlation among the vector components of the field, eventually its left- and righthanded projections.

We now adapt the paraxial model of Dennis [26] in order to account for the correlations intrinsic to a 2D light field. The key for explaining our results is that, in our system, the electric field can be modeled as a superposition of TE waves only. Note that we would find completely equivalent results considering the in-plane component of a field composed only of TM waves [54]. Contrarily, the coexistence of two independent TE and TM fields would add one extra degree of freedom to the system. In the unlikely scenario in which those two contributions have equal wavelength and amplitude for a given optical frequency, the results for the spatial correlation of $\mathrm{C}$ points would revert back to those observed in a $2 \mathrm{D}$ slice of a $3 \mathrm{D}$ field [54]. A TE mode in $2 \mathrm{D}$ can be expressed starting from a scalar field $H_{z}$ : 


$$
\begin{aligned}
& E_{x}=k_{y} H_{z} \\
& E_{y}=-k_{x} H_{z},
\end{aligned}
$$

which, by default, satisfies the transverse condition.

For a random wave field, we follow Berry's hypothesis and assume $H_{z}$ to be an isotropic superposition of monochromatic plane waves, each of them with a random phase $\delta_{\mathbf{k}}[53]$,

$$
H_{z}=\sum_{|\mathbf{k}|=k_{0}} \exp \left(i \mathbf{k} \cdot \mathbf{r}+i \delta_{\mathbf{k}}\right)
$$

where $\delta_{\mathbf{k}}$ is a random variable uniformly distributed in $[0,2 \pi]$. The autocorrelation of such a scalar, random, wave field is well known [53]: It is a Bessel function of order zero,

$$
C_{z z}(\mathbf{r})=\int d \mathbf{r}_{0} H_{z}^{*}\left(\mathbf{r}_{0}\right) H_{z}\left(\mathbf{r}_{0}+\mathbf{r}\right)=J_{0}\left(k_{0} r\right)
$$

The autocorrelations of $E_{x}$ and $E_{y}$ are also known [38], the main difference with $C_{z z}(\mathbf{r})$ being an anisotropic term dependent on the orientation $\varphi$ of $\mathbf{r}$ :

$$
\begin{aligned}
& C_{x x}(\mathbf{r})=\frac{1}{2}\left[J_{0}\left(k_{0} r\right)+\cos (2 \varphi) J_{2}\left(k_{0} r\right)\right], \\
& C_{y y}(\mathbf{r})=\frac{1}{2}\left[J_{0}\left(k_{0} r\right)-\cos (2 \varphi) J_{2}\left(k_{0} r\right)\right] .
\end{aligned}
$$

Also highly relevant to our study is the cross term among $E_{x}$ and $E_{y}$, which exhibits the following correlation:

$$
\begin{aligned}
C_{x y}(\mathbf{r}) & =\int d \mathbf{r}_{0} E_{x}^{*}\left(\mathbf{r}_{0}\right) E_{y}\left(\mathbf{r}_{0}+\mathbf{r}\right) \\
& =\frac{1}{2} \sin (2 \varphi) J_{2}\left(k_{0} r\right) .
\end{aligned}
$$

This equation can be easily proven by carrying out the integral in Fourier space and substituting the relations $E_{x}(\mathbf{k}) \propto \sin \left(\theta_{\mathbf{k}}\right) \delta\left(|\mathbf{k}|-k_{0}\right)$ and $E_{y}(\mathbf{k}) \propto-\cos \left(\theta_{\mathbf{k}}\right) \delta(|\mathbf{k}|-$ $\left.k_{0}\right)$ [38]. It is interesting to note that $E_{x}$ and $E_{y}$ only exhibit correlation when displaced since $C_{x y}(\mathbf{r})$ lacks the term proportional to $J_{0}$, and $J_{2}(0)=0$.

With these correlation functions known, and given the expression of $\psi_{l}$ and $\psi_{r}$ [Eq. (7)], we have all the ingredients to compute the correlations among the circular components of a TE random vector field. The autocorrelation of the left-handed component is

$$
\begin{aligned}
C_{l l}(\mathbf{r}) & =\int d \mathbf{r}_{0} \psi_{l}^{*}\left(\mathbf{r}_{0}\right) \psi_{l}\left(\mathbf{r}_{0}+\mathbf{r}\right) \\
& =C_{x x}(\mathbf{r})+C_{y y}^{*}(\mathbf{r})=J_{0}\left(k_{0} r\right),
\end{aligned}
$$

and similarly for $C_{r r}(\mathbf{r})$. The result of Eq. (13) is also identical to what we obtained in Eq. (10) for $H_{z}$, proving that each separate circular component behaves as a random scalar field. Similarly to Eq. (13), we can finally determine the correlation among left and right circular components:

$$
C_{l r}(\mathbf{r})=[\cos (2 \varphi)-i \sin (2 \varphi)] J_{2}\left(k_{0} r\right)
$$

and

$$
C_{r l}(\mathbf{r})=[\cos (2 \varphi)+i \sin (2 \varphi)] J_{2}\left(k_{0} r\right) .
$$

As elegantly explained by Berry and Dennis [53], the autocorrelation function of a complex field contains all the information needed to retrieve the pair and charge correlation function of its phase singularities. In the case of $\mathrm{C}$ points, i.e., phase singularities in the right- or left-handed field component, the cross terms $\left(C_{r l}\right.$ and $\left.C_{l r}\right)$ are also necessary. Following the same procedure of Berry and Dennis, we first calculate the point density of singularities in a scalar complex field, e.g., $\psi_{l} \equiv \psi_{l}^{\prime}+i \psi_{l}^{\prime \prime}$, which is defined as

$$
\rho\left[\mathbf{u}_{l}\right]=\delta\left(\psi_{l}^{\prime}\right) \delta\left(\psi_{l}^{\prime \prime}\right)\left|\frac{\partial \psi_{l}^{\prime}}{\partial x} \frac{\partial \psi_{l}^{\prime \prime}}{\partial y}-\frac{\partial \psi_{l}^{\prime}}{\partial y} \frac{\partial \psi_{l}^{\prime \prime}}{\partial x}\right|,
$$

where $\delta$ indicates the one-dimensional Dirac delta function and where, for compactness, we have introduced the real vector $\mathbf{u}_{l}=\left[\psi_{l}^{\prime}, \psi_{l}^{\prime \prime}, \partial_{x} \psi_{l}^{\prime}, \partial_{y} \psi_{l}^{\prime}, \partial_{x} \psi_{l}^{\prime \prime}, \partial_{y} \psi_{l}^{\prime \prime}\right]^{T}$. An analogous density can be defined for $\psi_{r}$.

The pair correlation function between $\mathrm{C}$ points at two different space points $\mathbf{r}_{A}$ and $\mathbf{r}_{B}$ and with opposite handedness can now be written in a straightforward way as

$$
g^{A}\left(\mathbf{r}_{B}-\mathbf{r}_{A}\right)=\frac{\left\langle\rho\left[\mathbf{u}_{l}\left(\mathbf{r}_{A}\right)\right] \rho\left[\mathbf{u}_{r}\left(\mathbf{r}_{B}\right)\right]\right\rangle}{\left\langle\rho\left[\mathbf{u}_{l}\left(\mathbf{r}_{A}\right)\right]\right\rangle\left\langle\rho\left[\mathbf{u}_{r}\left(\mathbf{r}_{B}\right)\right]\right\rangle} .
$$

In this equation, the notation $\left\langle f\left[\mathbf{u}_{l}\left(\mathbf{r}_{A}\right), \mathbf{u}_{r}\left(\mathbf{r}_{B}\right)\right]\right\rangle$ indicates the statistical average of a generic $f$, the functional of the field components and of their derivatives at different points in space. Introducing the combined vector $\mathbf{u}=\left[\mathbf{u}_{l}\left(\mathbf{r}_{A}\right), \mathbf{u}_{r}\left(\mathbf{r}_{B}\right)\right]^{T}$, the average can be explicitly written in the form

$$
\langle f[\mathbf{u}]\rangle=\frac{1}{(2 \pi)^{D / 2} \sqrt{\operatorname{det} M}} \int d^{D} \mathbf{u} f[\mathbf{u}] \exp \left(-\frac{1}{2} \mathbf{u}^{T} M^{-1} \mathbf{u}\right),
$$

where $D$ is the dimension of the vector $\mathbf{u}$ and $M$ is the matrix of the correlations between the various components of $\mathbf{u}$, i.e., $M_{i j}=\left\langle u_{i} u_{j}\right\rangle$. These elements correspond to the correlations between the different components of the left- and right-handed fields that we have summarized above, as well as their spatial derivatives. Similar expressions for different combinations of the fields $\psi_{l}$ and $\psi_{r}$ and for specific choices of the index of the singularities can be obtained from Eq. (17) with intuitive modifications. 
In some particular cases $[26,38,53]$, it is possible to derive a closed analytical expression for averages of the form in Eq. (17) by reducing the integrand to a quadratic form and integrating with standard mathematical techniques [55]. However, the specific form of the correlation matrix in our model does not lend itself easily to applying the formalism of Ref. [55]. This is because of the additional correlations between the real and imaginary parts of the field components, corresponding to the imaginary terms in $C_{l r}$ and $C_{r l}$ [Eqs. (14) and (15)]. Nevertheless, the average in Eq. (17) is particularly suited to numerical integration with Monte Carlo techniques [56]. We therefore calculate the pair correlation functions of $\mathrm{C}$ points and polarization vortices in two steps. First, we analytically perform the integral over the terms containing the Dirac delta functions in the integrand of Eq. (17). Subsequently, we numerically carry out the integration over the remaining variables, using the multidimensional Monte Carlo method [56]. We also validate our theoretical results by numerically simulating the superposition of random plane waves with TE polarization. The simulation results were all found in perfect agreement with the theoretical ones and are therefore not shown here.

We plot the theoretical expectations for the pair and charge correlation functions in direct comparison with the experimental data. In Fig. 4, we show the pair and charge correlation function for $\mathrm{C}$ points in 2D random vector waves and, in Fig. 6, the pair correlation functions for $\mathrm{C}$ points with the same or opposite handedness, respectively. For each of these curves, we find excellent agreement with the experiment. In particular, the pair correlation functions displayed in Fig. 6(b) for $\mathrm{C}$ points with opposite handedness represent the major novelty introduced by the model for 2D light. Among these functions, $g_{\text {same }}^{A}$ exhibits a behavior that is extremely unusual for pair correlations of this kind. Although this behavior is perfectly consistent with the experimental observation, it might conceal further interesting properties of random light confined in $2 \mathrm{D}$.

\section{CONCLUSIONS}

In this work, we investigated the spatial correlation of C points in $2 \mathrm{D}$ random light. We compared it to existing theory and experiments for 2D slices through a 3D random field in the paraxial regime. We demonstrated that confining the optical field to propagate in two dimensions induces severe changes in the spatial distribution of its $\mathrm{C}$ points. The shortage of degrees of freedom caused by the removal of one dimension results in a correlation among the vector components of the 2D light field. In the circular basis, this results in a correlation among the oppositely handed optical-spin components of light. One of the key consequences was the observation that the chance of finding $\mathrm{C}$ points with the same topological index actually increases as their mutual distance goes to zero. This is an unusual finding for dislocations of any kind. We quantify the correlation between left- and right-handed spin for the case of a TE field and incorporate it in a newly developed theoretical model. Our results are general for in-plane fields, including those of a TM mode as well. The outcome of the 2D model is found to be in perfect agreement with our experimental results. Given the unusual properties of the ensemble of $\mathrm{C}$ points in $2 \mathrm{D}$ random vector waves, our findings may trigger a reevaluation of concepts that are considered pillars of singular optics and topological defects, i.e., the sign principle [57] and topological screening [58]. Moreover, we anticipate that our results will trigger investigations of the evolution of $\mathrm{C}$ points as a function of external parameters, in order to explore phenomena such as same-index attraction and the formation of bound states among pairs of $\mathrm{C}$ points, which would be compatible with our observed correlation functions. Finally, the behavior of $\mathrm{C}$ points at short distances might lead to more unexplored features such as polarization vortices and higher-order singularities.

\section{ACKNOWLEDGMENTS}

We thank Andrea Di Falco for fabricating the chaotic cavity used in the near-field experiments and Thomas Bauer for useful discussions. Portions of this work were presented at the Frontiers in Optics in 2018. This work is part of the research program of the Netherlands Organization for Scientific Research (NWO). The authors acknowledge funding from the European Research Council (ERC Advanced Grant No. 340438-CONSTANS). F. A. acknowledges support from the Marie Skodowska-Curie individual fellowship BISTRO-LIGHT (Grant No. 748950).

[1] B. Hensen, H. Bernien, A. E. Dréau, A. Reiserer, N. Kalb, M. S. Blok, J. Ruitenberg, R. F. L. Vermeulen, R. N. Schouten, C. Abellán et al., Loophole-free Bell Inequality Violation Using Electron Spins Separated by 1.3 Kilometres, Nature (London) 526, 682 (2015).

[2] A. F. Koenderink, A. Alù, and A. Polman, Nanophotonics: Shrinking Light-based Technology, Science 348, 516 (2015).

[3] A. Polman, M. Knight, E. C. Garnett, B. Ehrler, and W. C. Sinke, Photovoltaic Materials: Present Efficiencies and Future Challenges, Science 352, aad4424 (2016).

[4] M. Burresi, R. J. P. Engelen, A. Opheij, D. van Oosten, D. Mori, T. Baba, and L. Kuipers, Observation of Polarization Singularities at the Nanoscale, Phys. Rev. Lett. 102, 033902 (2009).

[5] A. de Hoogh, L. Kuipers, T. Visser, and N. Rotenberg, Creating and Controlling Polarization Singularities in Plasmonic Fields, Photonics 2, 553 (2015).

[6] N. Rotenberg, B. le Feber, T. D. Visser, and L. Kuipers, Tracking Nanoscale Electric and Magnetic Singularities through Three-Dimensional Space, Optica 2, 540 (2015).

[7] B. Le Feber, N. Rotenberg, and L. Kuipers, Nanophotonic Control of Circular Dipole Emission, Nat. Commun. 6, 6695 (2015). 
[8] S.-H. Gong, F. Alpeggiani, B. Sciacca, E. C. Garnett, and L. Kuipers, Nanoscale Chiral Valley-Photon Interface through Optical Spin-Orbit Coupling, Science 359, 443 (2018).

[9] M. V. Berry, M. R. Dennis, and R. L. Lee Jr., Polarization Singularities in the Clear Sky, New J. Phys. 6, 162 (2004).

[10] G. Gbur, Singular Optics (CRC Press, Boca Raton, Florida, 2016).

[11] Y. Wang and G. Gbur, Hilbert's Hotel in Polarization Singularities, Opt. Lett. 42, 5154 (2017).

[12] G. Gbur, Fractional Vortex Hilbert's Hotel, Optica 3, 222 (2016).

[13] T. Fösel, V. Peano, and F. Marquardt, L Lines, C Points and Chern Numbers: Understanding Band Structure Topology Using Polarization Fields, New J. Phys. 19, 115013 (2017).

[14] A. Y. Bekshaev, K. Y. Bliokh, and F. Nori, Transverse Spin and Momentum in Two-Wave Interference, Phys. Rev. X 5, 011039 (2015).

[15] T. Bauer, M. Neugebauer, G. Leuchs, and P. Banzer, Optical Polarization Möbius Strips and Points of Purely Transverse Spin Density, Phys. Rev. Lett. 117, 013601 (2016).

[16] M. Antognozzi, C. R. Bermingham, R. L. Harniman, S. Simpson, J. Senior, R. Hayward, H. Hoerber, M. R. Dennis, A. Y. Bekshaev, K. Y. Bliokh et al., Direct Measurements of the Extraordinary Optical Momentum and Transverse SpinDependent Force Using a Nano-cantilever, Nat. Phys. 12, 731 (2016).

[17] K. Y. Bliokh, A. Y. Bekshaev, and F. Nori, Optical Momentum, Spin, and Angular Momentum in Dispersive Media, Phys. Rev. Lett. 119, 073901 (2017).

[18] K. Shibata, T. Tanigaki, T. Akashi, H. Shinada, K. Harada, K. Niitsu, D. Shindo, N. Kanazawa, Y. Tokura, and T.-h. Arima, Current-Driven Motion of Domain Boundaries between Skyrmion Lattice and Helical Magnetic Structure, Nano Lett. 18, 929 (2018).

[19] C. Reichhardt and C. J. Olson Reichhardt, Noise Fluctuations and Drive Dependence of the Skyrmion Hall Effect in Disordered Systems, New J. Phys. 18, 095005 (2016).

[20] A. Fert, N. Reyren, and V. Cros, Magnetic Skyrmions: Advances in Physics and Potential Applications, Nat. Rev. Mater. 2, 17031 (2017).

[21] T. N. Shendruk, A. Doostmohammadi, K. Thijssen, and J. M. Yeomans, Dancing Disclinations in Confined Active Nematics, Soft Matter 13, 3853 (2017).

[22] J. M. Yeomans, Active Matter: Playful Topology, Nat. Mater. 13, 1004 (2014).

[23] T. B. Saw, A. Doostmohammadi, V. Nier, L. Kocgozlu, S. Thampi, Y. Toyama, P. Marcq, C. T. Lim, J. M. Yeomans, and B. Ladoux, Topological Defects in Epithelia Govern Cell Death and Extrusion, Nature (London) 544, 212 (2017).

[24] A. Doostmohammadi, M. F. Adamer, S. P. Thampi, and J. M. Yeomans, Stabilization of Active Matter by FlowVortex Lattices and Defect Ordering, Nat. Commun. 7, 10557 (2016).

[25] M. V Berry and M. R Dennis, Polarization Singularities in Isotropic Random Vector Waves, Proc. R. Soc. A 457, 141 (2001).

[26] M. R. Dennis, Polarization Singularities in Paraxial Vector Fields: Morphology and Statistics, Opt. Commun. 213, 201 (2002).
[27] F. Flossmann, U. T. Schwarz, M. Maier, and M. R. Dennis, Polarization Singularities from Unfolding an Optical Vortex through a Birefringent Crystal, Phys. Rev. Lett. 95, 253901 (2005).

[28] F. Flossmann, K. O‘Holleran, M. R. Dennis, and M. J. Padgett, Polarization Singularities in $2 D$ and $3 D$ Speckle Fields, Phys. Rev. Lett. 100, 203902 (2008).

[29] K. O'Holleran, M. R. Dennis, and M. J. Padgett, Topology of Light's Darkness, Phys. Rev. Lett. 102, 143902 (2009).

[30] S. Barkhofen, J. J. Metzger, R. Fleischmann, U. Kuhl, and H.-J. Stöckmann, Experimental Observation of a Fundamental Length Scale of Waves in Random Media, Phys. Rev. Lett. 111, 183902 (2013).

[31] B. Redding, S. F. Liew, R. Sarma, and H. Cao, Compact Spectrometer Based on a Disordered Photonic Chip, Nat. Photonics 7, 746 (2013).

[32] T. Strudley, D. Akbulut, W. L. Vos, A. Lagendijk, A. P. Mosk, and O.L. Muskens, Observation of Intensity Statistics of Light Transmitted through 3D Random Media, Opt. Lett. 39, 6347 (2014).

[33] Z. Shi, M. Davy, and A. Z. Genack, Statistics and Control of Waves in Disordered Media, Opt. Express 23, 12293 (2015).

[34] D. Pierangeli, A. Tavani, F. Di Mei, A. J. Agranat, C. Conti, and E. DelRe, Observation of Replica Symmetry Breaking in Disordered Nonlinear Wave Propagation, Nat. Commun. 8, 1501 (2017).

[35] N. Bender, H. Yilmaz, Y. Bromberg, and H. Cao, Customizing Speckle Intensity Statistics, Optica 5, 595 (2018).

[36] S. N. Alperin and M. E. Siemens, Linear Model of Quantum Turbulent Vortex Dynamics, arXiv:1807.03899.

[37] C. Liu, R. E. C. van der Wel, N. Rotenberg, L. Kuipers, T. F. Krauss, A. Di Falco, and A. Fratalocchi, Triggering Extreme Events at the Nanoscale in Photonic Seas, Nat. Phys. 11, 358 (2015).

[38] L. De Angelis, F. Alpeggiani, A. Di Falco, and L. Kuipers, Spatial Distribution of Phase Singularities in Optical Random Vector Waves, Phys. Rev. Lett. 117, 093901 (2016).

[39] L. De Angelis, F. Alpeggiani, A. Di Falco, and L. Kuipers, Persistence and Lifelong Fidelity of Phase Singularities in Optical Random Waves, Phys. Rev. Lett. 119, 203903 (2017).

[40] G. R. Fowles, Introduction to Modern Optics (Dover Publications, New York, 1989).

[41] A. Mohtashami, C. I. Osorio, and A. F. Koenderink, Angle-Resolved Polarimetry of Antenna-Mediated Fluorescence, Phys. Rev. Applied 4, 054014 (2015).

[42] J. F. Nye, Lines of Circular Polarization in Electromagnetic Wave Fields, Proc. R. Soc. A 389, 279 (1983).

[43] M. R. Dennis, Polarization Singularity Anisotropy: Determining Monstardom, Opt. Lett. 33, 2572 (2008).

[44] B. A. Cvarch, B. Khajavi, J. A. Jones, B. Piccirillo, L. Marrucci, and E. J. Galvez, Monstar Polarization Singularities with Elliptically-Symmetric Q-plates, Opt. Express 25, 14935 (2017).

[45] J. A. Barker and D. Henderson, What Is "Liquid"? Understanding the States of Matter, Rev. Mod. Phys. 48, 587 (1976). 
[46] A. P. Lyubartsev and A. Laaksonen, Calculation of Effective Interaction Potentials from Radial Distribution Functions: A Reverse Monte Carlo Approach, Phys. Rev. E 52, 3730 (1995).

[47] M. Baldini, P. Postorino, L. Malavasi, C. Marini, K. W. Chapman, and H.-k. Mao, Pair Distribution Function Analysis: The Role of Structural Degrees of Freedom in the High-Pressure Insulator to Metal Transition of $\mathrm{VO}_{2}$, Phys. Rev. B 93, 245137 (2016).

[48] B. A. Frandsen, M. Brunelli, K. Page, Y. J. Uemura, J. B. Staunton, and S. J. L. Billinge, Verification of Anderson Superexchange in $\mathrm{MnO}$ via Magnetic Pair Distribution Function Analysis and ab initio Theory, Phys. Rev. Lett. 116, 197204 (2016).

[49] N. Thaicharoen, A. Schwarzkopf, and G. Raithel, Control of Spatial Correlations between Rydberg Excitations Using Rotary Echo, Phys. Rev. Lett. 118, 133401 (2017).

[50] J. M. Sobstyl, T. Emig, M. J. Abdolhosseini Qomi, F.-J. Ulm, and R. J.-M. Pellenq, Role of City Texture in Urban Heat Islands at Nighttime, Phys. Rev. Lett. 120, 108701 (2018).

[51] J.-P. Hansen and I. R. McDonald, Theory of Simple Liquids (Academic Press, London, 1990).

[52] H. Snijders, J. A. Frey, J. Norman, M. P. Bakker, E. C. Langman, A. Gossard, J. E. Bowers, M. P. Van Exter,
D. Bouwmeester, and W. Löffler, Purification of a SinglePhoton Nonlinearity, Nat. Commun. 7, 12578 (2016).

[53] M. V. Berry and M. R. Dennis, Phase Singularities in Isotropic Random Waves, Proc. R. Soc. A 456, 2059 (2000).

[54] See Supplemental Material at http://link.aps.org/ supplemental/10.1103/PhysRevX.8.041012 for the explanation of why considering a TE or TM mode would lead to equivalent statistics for its $\mathrm{C}$ points, and for the case of coexisting TE and TM modes.

[55] W. V. Li and A. Wei, Gaussian Integrals Involving Absolute Value Functions, in High Dimensional Probability V: The Luminy Volume, edited by C. Houdr, V. Koltchinskii, D. M. Mason, and M. Peligrad (Institute of Mathematical Statistics, Beachwood, Ohio, 2009), Vol. 5, pp. 43-59.

[56] W. H. Press, S. A. Teukolsky, W. T. Vetterling, and B. P. Flannery, Numerical Recipes: The Art of Scientific Computing, 3rd ed. (Cambridge University Press, New York, 2007).

[57] I. Freund and N. Shvartsman, Wave-Field Phase Singularities: The Sign Principle, Phys. Rev. A 50, 5164 (1994).

[58] I. Freund and M. Wilkinson, Critical-Point Screening in Random Wave Fields, J. Opt. Soc. Am. A 15, 2892 (1998). 